01,13

\title{
Моделирование радиационно-индуцированной сегрегации в сплавах $\mathrm{Fe}-\mathrm{Cr}-\mathrm{Ni}$
}

\author{
(C) Р.В. Скороход, А.В. Коропов
}

Институт прикладной фризики НАН Украины, Сумы, Украина

E-mail: r.skorokhodqq@gmail.com

Поступила в Редакцию 16 июля 2019 г.

В окончательной редакции 16 июля 2019 г.

Принята к публикации 25 июля 2019 г.

В модели радиационно-индуцированной сегрегации, базирующейся на первом и втором законах Фика и учитывающей обратный эффект Киркендалла, получены концентрационные профили компонентов концентрированного сплава Fe-Cr-Ni и радиационных точечных дефектов при различных температурах, плотностях дислокаций, скоростях набора дозы. Расчет концентрационных профилей проведен от момента начала облучения до выхода на стационарный режим. Проанализирована чувствительность концентраций компонентов сплава вблизи поверхности от входных параметров (энергий миграции вакансий $\mathrm{Cr}, \mathrm{Ni}, \mathrm{Fe}$, энергии миграции межузельных атомов и др.).

Ключевые слова: радиационно-индуцированная сегрегация, концентрированные металлические сплавы, концентрационные профили, точечные дефекты, компьютерное моделирование.

DOI: $10.21883 /$ FTT.2019.12.48605.53ks

\section{1. Введение}

Радиационно-индуцированные явления в металлических сплавах, как правило, приводят к ухудшению механических и физико-химических свойств конструкционных материалов ядерных реакторов (см., например, [13]). К радиационно-индуцированным явлениям относится и явление радиационно-индуцированной сегрегации (РИС), которое заключается в пространственном перераспределении компонентов металлического сплава под действием облучения при промежуточных температу$\operatorname{pax}\left(0.3-0.5 T_{\text {пл }}\right)$. Результатом РИС является обогащение или обеднение образца по основным компонентам сплава, легирующим или примесным элементам вблизи стоков дефектов (свободных поверхностей, дислокаций, межзеренных границ и др.) [1]. Такие локальные изменения концентраций компонентов металлического сплава, в частности, могут привести к возникновению выделений новой фазы $[4,5]$ и к ухудшению механической прочности межзеренных границ (охрупчивание материала по межзеренным границам) [2]. Особенно важным является изучение РИС в современных корпусных материалах ядерных реакторов, которые за длительный срок эксплуатации получают высокие дозы радиационных повреждений (более 40 смещений на атом) и при этом должны сохранять необходимые механические и физикохимические свойства и стабильные размеры [2].

Для моделирования РИС был выбран трехкомпонентный концентрированный сплав $\mathrm{Fe}-\mathrm{Cr}-\mathrm{Ni}$, концентрационные изменения в котором хорошо описывает модель, базирующаяся на первом и втором законах Фика и учитывающая обратный эффект Киркендалла [1,6]. Компоненты выбранного сплава являются основными компонен- тами большинства реальных нержавеющих сталей, которые обычно содержат большее количество компонентов и используются как материалы ядерной энергетики [2].

Целью данной работы является расчет концентрационных профилей компонентов трехкомпонентного концентрированного сплава $\mathrm{Fe}-\mathrm{Cr}-\mathrm{Ni}$ и радиационных точечных дефектов (ТД) в нем под действием облучения при различных его дозах в широком диапазоне значений температур, плотностей дислокаций и скоростей набора дозы.

\section{2. Постановка задачи}

Далее будем моделировать РИС в образце сплава $\mathrm{Fe}-20 \% \mathrm{Cr}-8 \% \mathrm{Ni}$ (состав сплава задан в атомных процентах), представляющего собой слой с постоянной толщиной $\ell$ и занимающего область $0 \leq x \leq \ell$. Считается, что стоками ТД являются как поверхности слоя, так и дислокации, входящие в состав дислокационной подсистемы образца. Тогда пространственно-временная эволюция концентраций вакансий $X_{v}(x, t)$, межузельных атомов $X_{i}(x, t)$ и компонентов рассматриваемого сплава $\left(X_{\mathrm{Fe}}, X_{\mathrm{Cr}}, X_{\mathrm{Ni}}\right)$ задается системой четырех связанных нелинейных дифференциальных уравнений в частных производных [1,7]:

$$
\left\{\begin{array}{l}
\frac{\partial X_{v}}{\partial t}=-\nabla \mathbf{J}_{v}+K_{0}-R_{i v} X_{v} X_{i}-R_{v D}\left(X_{v}-X_{v}^{e q}\right), \\
\frac{\partial X_{i}}{\partial t}=-\nabla \mathbf{J}_{i}+K_{0}-R_{i v} X_{v} X_{i}-R_{i D}\left(X_{i}-X_{i}^{e q}\right), \\
\frac{\partial X_{k}}{\partial t}=-\nabla \mathbf{J}_{k},
\end{array}\right.
$$

где $\mathbf{J}_{m}$ - потоки атомов и ТД сорта $m, K_{0}-$ скорость набора дозы, $R_{i v}$ - коэффициент рекомбинации ТД, $R_{v D}$ 
и $R_{i D}$ - скорости поглощения дислокациями соответственно вакансий и межузельных атомов, $X_{v}^{e q}$ и $X_{i}^{e q}-$ термодинамически равновесные концентрации ТД. Коэффициент рекомбинации $R_{i v}$ в системе уравнений (1) определяется формулой [8]:

$$
R_{i v}=\frac{4 \pi r_{i v}}{\Omega}\left[\sum_{k=\mathrm{Fe}, \mathrm{Cr}, \mathrm{Ni}}\left(d_{k, v}+d_{k, i}\right) X_{k}\right],
$$

где $r_{i v}$ - радиус рекомбинации, $\Omega-$ средний объем, приходящийся на один атом в сплаве, $d_{k, d}$ (здесь и далее нижний индекс $d$ принимает буквенные значения $v$ или $i)$ - кинетические коэффициенты, связанные с парциальными коэффициентами диффузии атомов и ТД [1]. Поскольку сплав $\mathrm{Fe}-20 \% \mathrm{Cr}-8 \% \mathrm{Ni}$ имеет ГЦК решетку, средний объем, приходящийся на один атом, равен $\Omega=a^{3} / 4$, где $a-$ параметр решетки. Скорости поглощения дислокациями вакансий $R_{v D}$ и межузельных атомов $\rho_{i D}$ задаются выражениями [1]:

$$
R_{d D}=4 \pi r_{d D}\left(\sum_{k=\mathrm{Fe}, \mathrm{Cr}, \mathrm{Ni}} d_{k, d} X_{k}\right) \frac{\rho_{D}}{\Delta},
$$

где $r_{d D}$ - радиус захвата дислокацией ТД сорта $d, \rho_{D}-$ плотность дислокаций в дислокационной подсистеме, $\Delta$ - межплоскостное расстояние. Радиус захвата ТД $r_{d D}$ имеет порядок нескольких параметров решетки; в данной работе, как и в работе [9], принято $r_{d D}=1 \cdot 10^{-9} \mathrm{~m}$.

Заметим, что в системе уравнений (1) концентрации ТД и компонентов сплава выражены в молярных долях. Учитывая то, что $X_{v}$ и $X_{i}$ обычно на несколько порядков меньше концентраций компонентов сплава, с высокой степенью точности имеем равенство $X_{\mathrm{Fe}}+X_{\mathrm{Cr}}+X_{\mathrm{Ni}}=1$.

Потоки вакансий $\mathbf{J}_{v}$, межузельных атомов $\mathbf{J}_{i}$ и компонентов сплава $\mathbf{J}_{k}$ в рамках общепринятой теории РИС [1] равны

$$
\begin{aligned}
\mathbf{J}_{v} & =-\sum_{k=\mathrm{Fe}, \mathrm{Cr}, \mathrm{Ni}} d_{k, v} X_{k} \nabla X_{v}+\alpha X_{v}\left(\sum_{k=\mathrm{Fe}, \mathrm{Cr}, \mathrm{Ni}} d_{k, v} \nabla X_{v}\right) \\
\mathbf{J}_{i} & =-\sum_{k=\mathrm{Fe}, \mathrm{Cr}, \mathrm{Ni}} d_{k, i} X_{k} \nabla X_{i}-\alpha X_{i}\left(\sum_{k=\mathrm{Fe}, \mathrm{Cr}, \mathrm{Ni}} d_{k, i} \nabla X_{k}\right) \\
\mathbf{J}_{k} & =-\left(\sum_{d=v, i} d_{k, d} X_{d}\right) \alpha \nabla X_{k}+X_{k}\left(d_{k, v} \nabla X_{v}-d_{k, i} \nabla X_{i}\right)
\end{aligned}
$$

где $\alpha-$ термодинамический фактор (в данной работе принято $\alpha=1$ ).

Для кинетических коэффициентов $\left(d_{k, v}\right.$ и $\left.d_{k, i}\right)$ имеют место выражения [1]:

$$
\begin{aligned}
d_{k, v} & =\frac{1}{6} z_{v} f_{k, v} \lambda_{v}^{2} \omega_{k, v}, \\
d_{k, i} & =\frac{1}{6} z_{i} f_{k, i} \lambda_{i}^{2} \omega_{k, i},
\end{aligned}
$$

где $z_{d}-$ количество ближайших соседей (координационное число) для ТД сорта $d, f_{k, d}-$ корреляционный фактор. Расстояние от ТД до ближайшего соседа $\lambda_{d}$ можно записать как $\lambda_{d}=A_{d} a$, где $A_{d}-$ безразмерная константа, определяемая типом кристаллической решетки. Количество ближайших соседей $z_{d}$ и безразмерные константы $A_{d}$ для ГЦК-решетки соответственно равны $z_{v}=z_{i}=12, A_{v}=1 / \sqrt{2}$ и $A_{i}=1 / 2$. Частоты перескоков вакансий $\omega_{k, v}$ и межузельных атомов $\omega_{k, i}$ для элемента сорта $k$ могут быть представлены в следующем виде [1]:

$$
\begin{aligned}
\omega_{k, v} & =\omega_{k, v}^{0} \exp \left(\frac{-E_{k, v}^{m}}{k_{b} T}\right), \\
\omega_{k, i} & =\omega_{k, i}^{0} \exp \left(\frac{-E_{k, i}^{m}}{k_{b} T}\right),
\end{aligned}
$$

где $\omega_{k, d}^{0}-$ предэкспоненциальный фактор, $T-$ температура, $k_{b}$ - постоянная Больцмана, $E_{k, d}^{m}-$ энергия миграции ТД посредством атома сорта $k$.

Используя равенства (7)-(10), выражения для кинетических коэффициентов $d_{k, d}$ можно записать в аррениусовской форме

$$
\begin{aligned}
d_{k, v} & =d_{k, v}^{0} \exp \left(\frac{-E_{k, v}^{m}}{k_{b} T}\right), \\
d_{k, i} & =d_{k, i}^{0} \exp \left(\frac{-E_{k, i}^{m}}{k_{b} T}\right),
\end{aligned}
$$

где множители $d_{k, v}^{0}$ и $d_{k, i}^{0}$ имеют вид

$$
\begin{aligned}
d_{k, v}^{0} & =\frac{1}{6} z_{v} f_{k, v} A_{v}^{2} a^{2} \omega_{k, v}^{0}, \\
d_{k, i}^{0} & =\frac{1}{6} z_{i} f_{k, i} A_{i}^{2} a^{2} \omega_{k, i}^{0} .
\end{aligned}
$$

Будем считать, что до облучения (в начальный момент времени $t=0$ ) атомы сплава распределены случайно по всему образцу

$$
\begin{gathered}
\left.X_{\mathrm{Fe}}(x, t)\right|_{t=0}=0.72,\left.\quad X_{\mathrm{Cr}}(x, t)\right|_{t=0}=0.20, \\
\left.X_{\mathrm{Ni}}(x, t)\right|_{t=0}=0.08 .
\end{gathered}
$$

В качестве дополнительных начальных условий принимаем, что начальные концентрации ТД равны термодинамически равновесным $\left(X_{v}^{e q}, X_{i}^{e q}\right)$

$$
\begin{gathered}
\left.X_{v}(x, t)\right|_{t=0}=X_{v}^{e q}=\exp \left(\frac{S_{v}^{f}}{k_{b}}\right) \exp \left(-\frac{E_{\mathrm{Fe}, v}^{f}}{k_{b} T}\right), \\
\left.X_{i}(x, t)\right|_{t=0}=X_{i}^{e q}=\exp \left(-\frac{E_{\mathrm{Fe}, i}^{f}}{k_{b} T}\right),
\end{gathered}
$$

где $S_{v}^{f}$ - энтропия формирования вакансий, $E_{\mathrm{Fe}, v}^{f}-$ энергия формирования вакансий $\mathrm{Fe}, E_{\mathrm{Fe}, i}^{f}-$ энергия формирования межузельных атомов $\mathrm{Fe}$.

В качестве граничных условий к системе уравнений (1) принимаем следующие. Граничные условия на левой поверхности слоя $(x=0)$ таковы

$$
\left.X_{v}(x, t)\right|_{x=0}=X_{v}^{e q},\left.\quad X_{i}(x, t)\right|_{x=0}=X_{i}^{e q} .
$$


Входные параметры

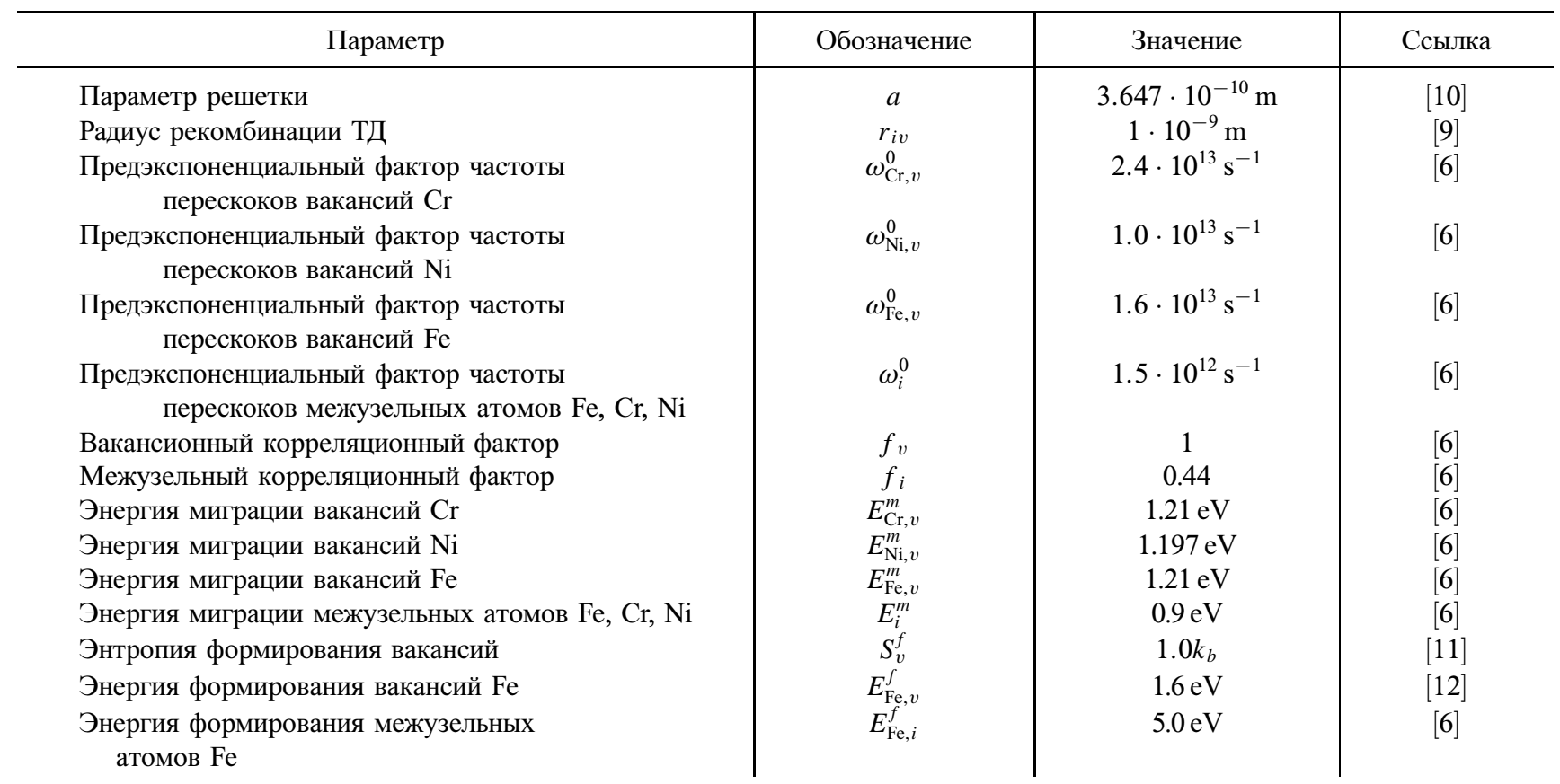

Далее считаем, что задача симметрична относительно плоскости $x=\ell / 2$, а в плоскости симметрии градиенты всех концентраций равны нулю

$$
\begin{aligned}
\left.\frac{\partial X_{v}(x, t)}{\partial x}\right|_{x=\ell / 2} & =\left.\frac{\partial X_{i}(x, t)}{\partial x}\right|_{x=\ell / 2} \\
& =\left.\frac{\partial X_{k}(x, t)}{\partial x}\right|_{x=\ell / 2}=0 .
\end{aligned}
$$

Поскольку атомы вещества не имплантируются в образец и не удаляются из него, для концентраций атомов сплава $X_{k}(x, t)$ справедливы законы сохранения вещества

$$
\int_{0}^{\ell / 2} X_{k}(x, t) d x=\left.\frac{\ell}{2} X_{k}(x, t)\right|_{t=0},
$$

которые играют роль дополнительных граничных условий.

Система уравнений (1) с начальными и граничными условиями (15)-(20) решалась численно. Входные параметры, использованные при решении системы (1), представлены в таблице. Математический алгоритм решения аналогичен тому, который был использован в работе [13]. Все расчеты проведены для образцов сплава $\mathrm{Fe}-20 \% \mathrm{Cr}-8 \% \mathrm{Ni}$ толщиной $\ell=400 \cdot 10^{-9} \mathrm{~m}$ с шагом разностной схемы $0.3333 \cdot 10^{-9} \mathrm{~m}$ по пространственной координате $x$. Значения параметров, если не оговорено иное, при моделировании считались следующими: температура $T=350^{\circ} \mathrm{C}$, плотность дислокаций $\rho_{D}=10^{14} \mathrm{~m}^{-2}[6]$, доза облучения $D=10 \mathrm{dpa}$ (displacements per atom), скорость набора дозы $K_{0}=10^{-6} \mathrm{dpa} / \mathrm{s}$.

\section{3. Результаты и обсуждения}

Чувствительность модели к входным параметрам аппроксимируем как $[6,13]$

$$
\frac{\partial X}{\partial p} \approx \frac{\delta X}{\delta p}=\frac{X^{\prime}-X_{\text {reference }}}{p^{\prime}-p_{\text {reference }}},
$$

где $X_{\text {reference }}$ - значение концентрации в непосредственной близости к поверхности образца $(x \rightarrow+0)$, рассчитанное при эталонном значении входного параметра $p_{\text {reference }}, X^{\prime}-$ значение концентрации в непосредственной близости к поверхности образца, рассчитанное при значении входного параметра $p^{\prime}$, для которых должны выполнятся следующие условия

$$
\begin{aligned}
& \left|p^{\prime}-p_{\text {reference }}\right| \ll p_{\text {reference }}, \\
& \left|X^{\prime}-X_{\text {reference }}\right| \ll X_{\text {reference }} .
\end{aligned}
$$

Как и в работах $[6,13]$, от чувствительности удобно перейти к безразмерной характеристике - значимости

$$
S_{p}^{X}=\frac{X^{\prime}-X_{\text {reference }}}{p^{\prime}-p_{\text {reference }}} \frac{p_{\text {reference }}}{X_{\text {reference }}} .
$$

Из выражения (24) следует, что концентрации компонентов сплава в непосредственной близости к поверхности образца наиболее чувствительны к тем параметрам, которые имеют наибольшие абсолютные значения значимостей. При этом положительное значение значимости отвечает увеличению концентрации с ростом значения соответствующего параметра, а отрицательное - уменьшению. 

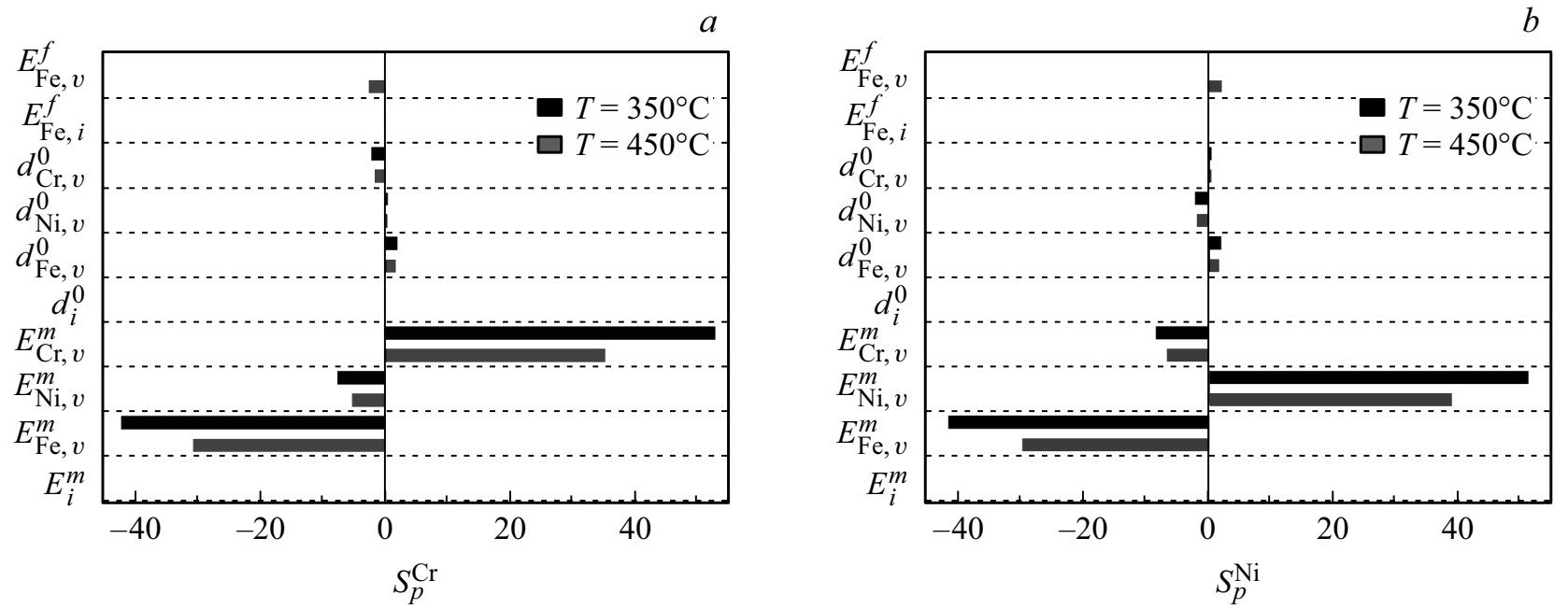

Рис. 1. Значимости параметров для концентраций $\mathrm{Cr}(a)$ и $\mathrm{Ni}(b)$, рассчитанные в непосредственной близости к поверхности образца.
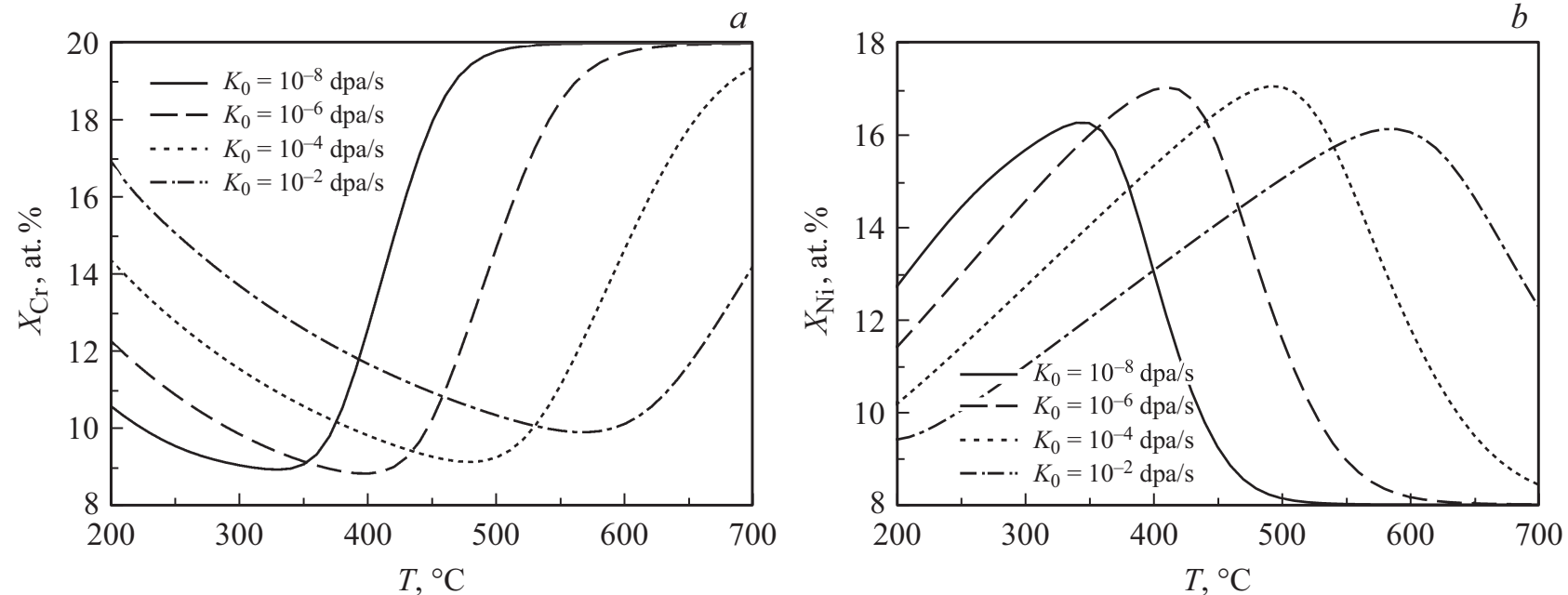

Рис. 2. Зависимости концентраций $\mathrm{Cr}(a)$ и Ni $(b)$ от температуры $T$, рассчитанные при различных скоростях набора дозы $K_{0}$.
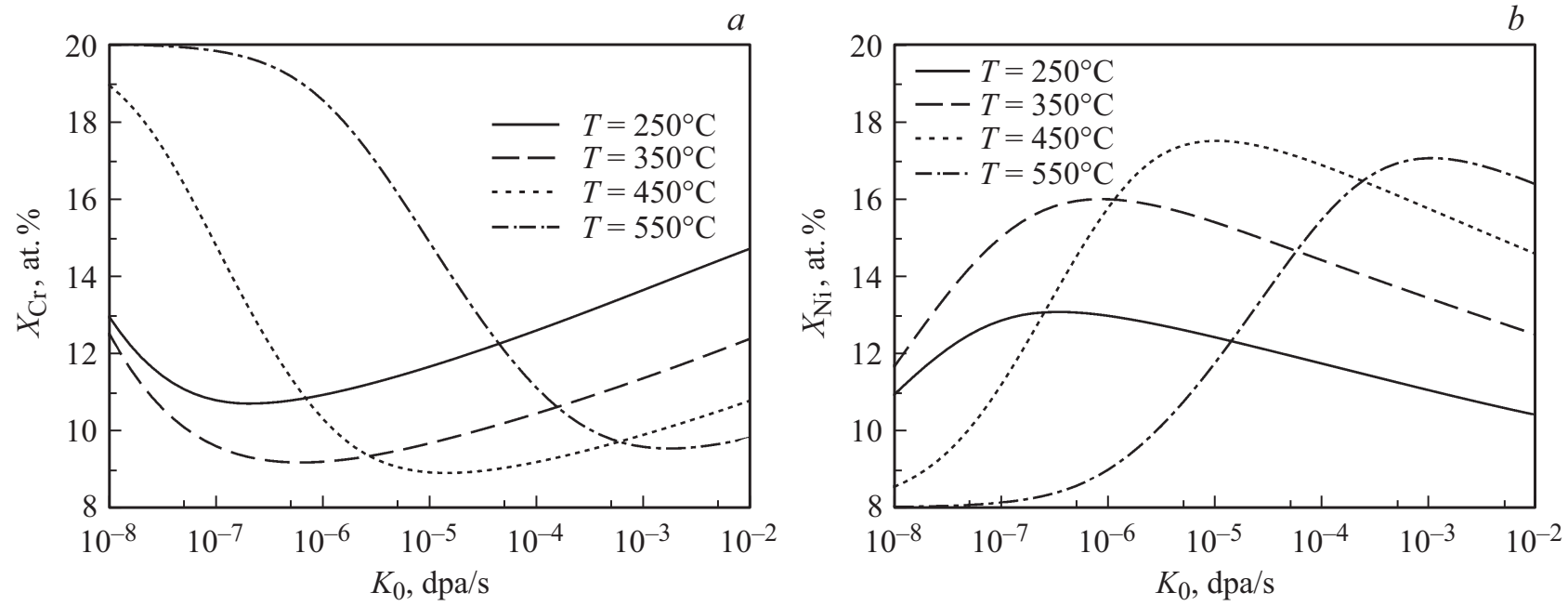

Рис. 3. Зависимости концентраций $\mathrm{Cr}(a)$ и $\mathrm{Ni}(b)$ от скорости набора дозы $K_{0}$, рассчитанные при различных температурах $T$. 


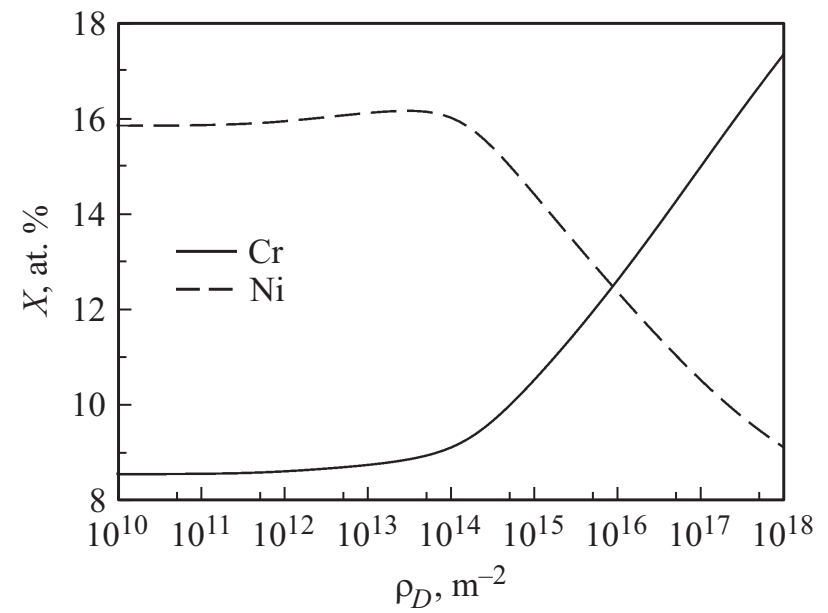

Рис. 4. Зависимость концентрации $\mathrm{Cr}$ и $\mathrm{Ni}$ от плотности дислокаций $\rho_{D}$.

Значимости параметров $S_{p}^{\mathrm{Cr}}$ и $S_{p}^{\mathrm{Ni}}$ были рассчитаны при увеличении входных параметров на $0.1 \%$ для температур 350 и $450^{\circ} \mathrm{C}$; соответствующие данные приведены на рис. 1. Концентрация $\mathrm{Cr}$ наиболее существенно зависит от энергий миграции вакансий $\mathrm{Cr}\left(E_{\mathrm{Cr}, v}^{m}\right)$ и $\mathrm{Fe}\left(E_{\mathrm{Fe}, v}^{m}\right)$, немного слабее от энергии миграции вакансий $\mathrm{Ni}\left(E_{\mathrm{Ni}, v}^{m}\right)$ и практически не зависит от энергии миграции межузельных атомов $E_{i}^{m}$ (как следует из таблицы, энергии миграции межузельных атомов $\mathrm{Fe}$, $\mathrm{Cr}$, Ni считаются одинаковыми). C увеличением энергий миграции вакансий $\mathrm{Cr}$ концентрация $\mathrm{Cr}$ увеличивается, a c ростом энергий миграции вакансий $\mathrm{Ni}$ и $\mathrm{Fe}-$ уменьшается. Для зависимостей концентрации $\mathrm{Ni}$ от энергий миграции вакансий наблюдается ситуация, аналогичная зависимостям концентрации Сr. А именно, концентрация Ni наиболее существенно зависит от энергий миграции вакансий $\mathrm{Ni}$ и $\mathrm{Fe}$, немного слабее от энергии миграции вакансий $\mathrm{Cr}$ и практически не зависит от энергии миграции межузельных атомов. В сравнении с работой [13], где моделировался двухкомпонентный концентрированный сплав $\mathrm{Fe}-\mathrm{Cr}$, в данной работе абсолютные значения значимостей параметров $S_{p}^{X}$ более слабо уменьшаются с повышением температуры $T$, а для энергии формирования вакансий $\mathrm{Fe}\left(E_{\mathrm{Fe}, v}^{f}\right)$ наблюдается рост абсолютного значения значимости с повышением температуры.

Переходя к обсуждению полученных зависимостей, отметим, что все зависимости, представленные на рис. 2-5, относятся к непосредственной близости поверхности образца.

На рис. 2 показаны зависимости $X_{\mathrm{Cr}}=X_{\mathrm{Cr}}(T)$ и $X_{\mathrm{Ni}}=X_{\mathrm{Ni}}(T)$ при различных значениях $K_{0}$. Для всего интервала значений температур $\left(200<T<700^{\circ} \mathrm{C}\right)$ наблюдаются обеднение по $\mathrm{Cr}$ и обогащение по $\mathrm{Ni}$. Рассмотрим данные зависимости для скорости набора дозы $K_{0}=10^{-6} \mathrm{dpa} / \mathrm{s}$ (см. штриховые кривые на рис. 2, $a$ и $b$ ). При температуре $T=397^{\circ} \mathrm{C}$ концентрация $\mathrm{Cr}$ принимает минимальное значение, равное $8.84 \%$. В дальнейшем с увеличением температуры наблюдается увеличение концентрации $\mathrm{Cr}$ до $20 \%$, что обусловлено увеличением коэффициента рекомбинации и ростом концентрации термодинамически равновесных ТД $X_{v}^{e q}$ и $X_{i}^{e q}$. Максимальное значение обогащения $\mathrm{Ni}$, равное $17.03 \%$, наблюдается при температуре $T=412^{\circ} \mathrm{C}$. Дальнейшее уменьшение концентрации $\mathrm{Ni}$ (как и увеличение концентрации $\mathrm{Cr}$ для температур $T>397^{\circ} \mathrm{C}$ ) объясняется увеличением обратной диффузии с ростом температуры. Аналогичная ситуация наблюдается и для других скоростей набора дозы $K_{0}=10^{-8} \mathrm{dpa} / \mathrm{s}, K_{0}=10^{-4} \mathrm{dpa} / \mathrm{s}$, и $K_{0}=10^{-2} \mathrm{dpa} / \mathrm{s}$. При этом температура максимума обеднения по $\mathrm{Cr}$ и обогащения по $\mathrm{Ni}$ сдвигается в область более высоких температур, что можно объяснить увеличением количества неравновесных ТД с ростом скорости набора дозы (см. рис. 6, $e$ и $f$ ).

На рис. 3 приведены зависимости $X_{\mathrm{Cr}}=X_{\mathrm{Cr}}\left(K_{0}\right)$ и $X_{\mathrm{Ni}}=X_{\mathrm{Ni}}\left(K_{0}\right)$ при различных значениях $T$. С ростом температуры минимальное значение концентрации $\mathrm{Cr}$ и
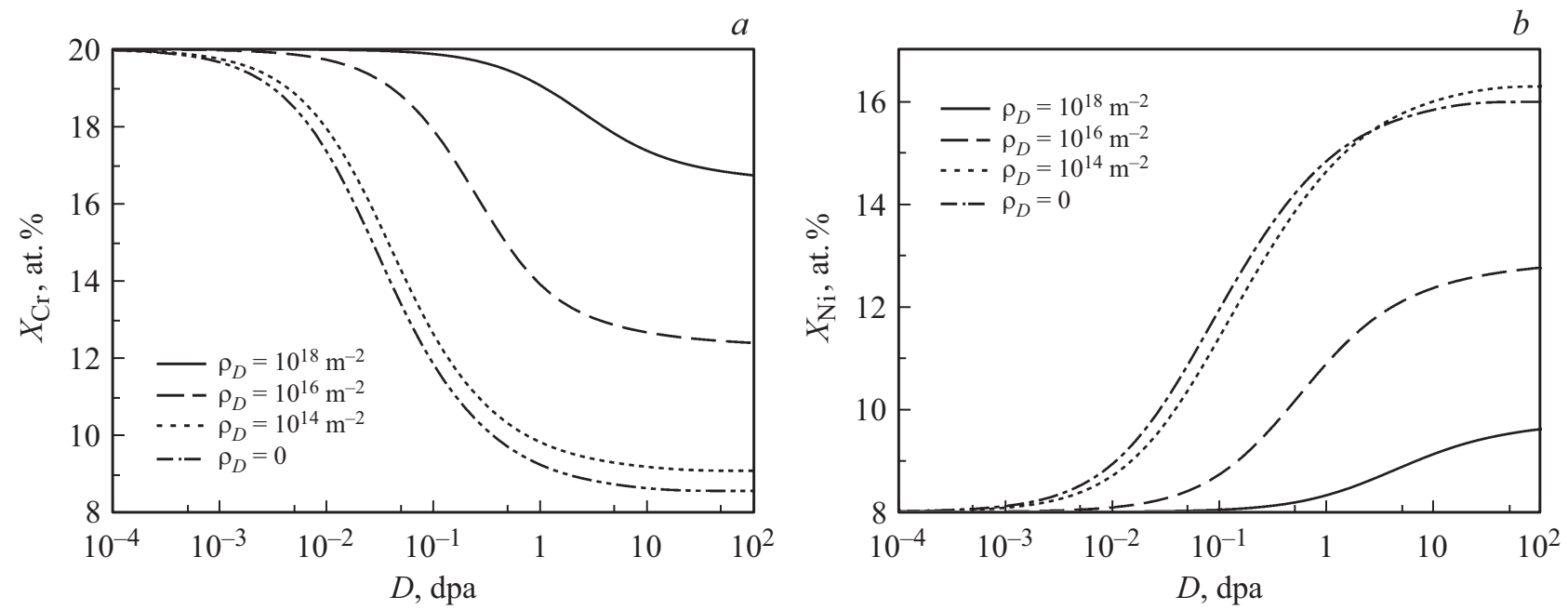

Рис. 5. Зависимости концентраций $\mathrm{Cr}(a)$ и $\mathrm{Ni}(b)$ от набранной дозы $D$, рассчитанные при различных плотностях дислокаций $\rho_{D}$. 

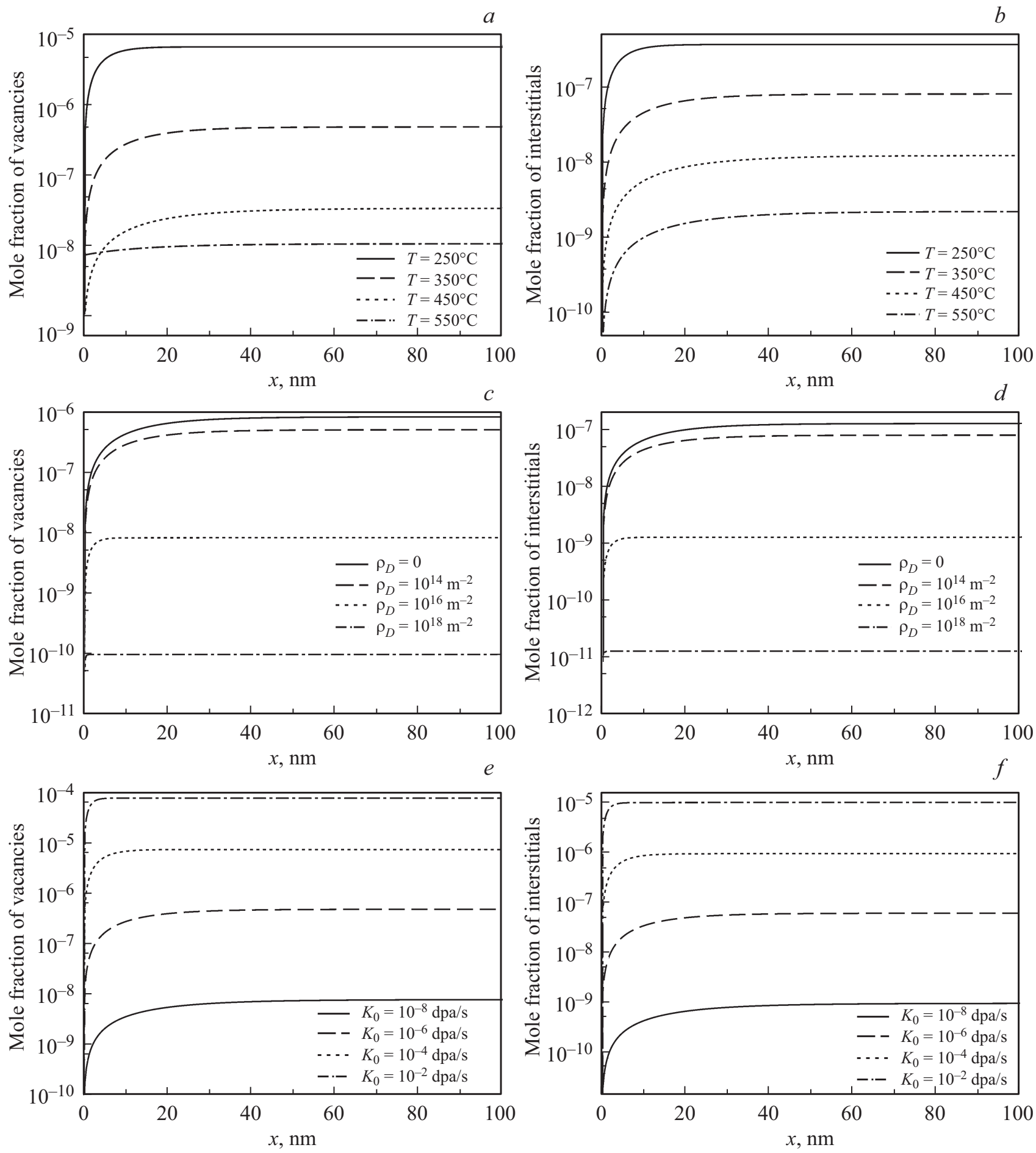

Рис. 6. Концентрационные профили вакансий и межузельных атомов, рассчитанные при различных температурах $T(a, b)$, плотностях дислокаций $\rho_{D}(c, d)$ и скоростях набора дозы $K_{0}(e, f)$.

максимальное значение концентрации $\mathrm{Ni}$ сдвигаются в область более высоких скоростей набора дозы $K_{0}$.

Зависимости концентраций $\quad X_{\mathrm{Cr}}=X_{\mathrm{Cr}}\left(\rho_{D}\right) \quad$ и $X_{\mathrm{Ni}}=X_{\mathrm{Ni}}\left(\rho_{D}\right)$ при температуре $T=350^{\circ} \mathrm{C}$, скорости набора дозы $K_{0}=10^{-6} \mathrm{dpa} / \mathrm{s}$ и дозе облучения $D=10 \mathrm{dpa}$ представлены на рис. 4. Как видно, наблюдается рост значения концентрации $\mathrm{Cr}$ с увеличением плотности дислокаций. Переход от достаточно слабого роста к более сильному наблюдается при $\rho_{D} \gtrsim 10^{14} \mathrm{~m}^{-2}$. Зависимость концентрации $\mathrm{Ni}$ от плотности дислокаций имеет слабо выраженный максимум при $\rho_{D} \approx 3 \cdot 10^{13} \mathrm{~m}^{-2}$. Поскольку дислокации являются стоками ТД, с ростом плотности дислокаций $\rho_{D}$ уменьшается концентрация ТД, что при достаточно 
высоких значениях $\rho_{D} \quad\left(\rho_{D} \gtrsim 10^{14} \mathrm{~m}^{-2}\right)$ приводит к ослаблению эффектов сегрегации вблизи свободной поверхности образца (концентрации $\mathrm{Cr}$ и $\mathrm{Ni}$ меньше отличаются от начальных значений (15)).

На рис. 5 приведены зависимости $X_{\mathrm{Cr}}=X_{\mathrm{Cr}}(D)$ и $X_{\mathrm{Ni}}=X_{\mathrm{Ni}}(D)$ при различных значениях $\rho_{D}$. С ростом дозы облучения уменьшается концентрация $\mathrm{Cr}$ и увеличивается концентрация $\mathrm{Ni}$ при всех рассмотренных значениях плотности дислокаций. Как видно из рис. 5, достаточно сильное влияние дислокационной подсистемы на концентрационные зависимости проявляется при $\rho_{D} \gtrsim 10^{14} \mathrm{~m}^{-2}$. Как следует из расчетов, с увеличением плотности дислокаций возрастает значение дозы, при которой происходит выход на стационарный режим.

На рис. 6 представлены зависимости концентраций ТД от координаты $x$. Поскольку межузельные атомы диффундируют намного быстрее вакансий, концентрации межузельных атомов значительно меныше концентраций вакансий при одинаковых условиях облучения (температурах $T$, плотностях дислокаций $\rho_{D}$ и скоростях набора дозы $\left.K_{0}\right)$, хотя они, как правило, имеют подобную форму. Как видно из рис. 6, $a, b$, при увеличении температуры уменьшаются концентрации радиационных ТД за счет их более быстрой диффузии на стоки и увеличения коэффициента рекомбинации ТД. Так, уже при температуре $T=550^{\circ} \mathrm{C}$ концентрация радиационных вакансий в глубине образца $(x \gtrsim 20 \mathrm{~nm})$ слабо отличается от их термодинамически равновесной концентрации $X_{v}^{e q}=7.26 \cdot 10^{-9}$ (см. штрихпунктирную кривую на рис. $6, a)$. Также концентрации радиационных ТД уменьшаются с увеличением значения плотности дислокаций $\rho_{D}$ (см. рис. $6, c$ и $d$ ), поскольку дислокации являются стоками ТД внутри образца. Отметим, что значения концентраций как вакансий, так и межузельных атомов при плотностях дислокаций $\rho_{D}=0$ и $\rho_{D}=10^{14} \mathrm{~m}^{-2}$ практически не отличаются. С ростом скорости набора дозы $K_{0}$ увеличиваются концентрации радиационных ТД (см. рис. 6, $е$ и $f$ ).

В общем случае многокомпонентного концентрированного сплава обогащение или обеднение по компонентам сплава вблизи стоков дефектов в стационарном режиме определяется знаком дискриминанта РИС $M_{k}[1,14]$ :

$M_{k}=\frac{\left(d_{k, v} X_{k} / D_{k}\right) \sum_{m \neq k} d_{m, i} X_{m} / D_{m}-\left(d_{k, i} X_{k} / D_{k}\right) \sum_{m \neq k} d_{m, v} X_{m} / D_{m}}{\alpha \sum_{m} d_{m, i} X_{m} / D_{m}}$,

$$
\nabla X_{k}=M_{k} \nabla X_{v} .
$$

В формуле (25) $D_{k}-$ коэффициенты диффузии атомов сорта $k$, для которых справедливо равенство $D_{k}=d_{k, v} X_{v}+d_{k, i} X_{i}$ (см., например, [1]). Из формулы (26) следует, что при $M_{k}>0$ векторы $\nabla X_{k}$ и $\nabla X_{v}$ направлены в одну сторону, а при $M_{k}<0-$ в противоположные стороны. Тогда при $M_{k}>0$ будет наблюдаться обеднение по элементу $k$ вблизи стоков ТД, поскольку концентрация вакансий уменьшается в направлении стоков ТД (см. рис. 6, a,c,e). При $M_{k}<0$, напротив, будет наблюдаться эффект обогащения. Для рассматриваемого сплава $\mathrm{Fe}-20 \% \mathrm{Cr}-8 \% \mathrm{Ni}$ при темпеpaтуре $T=350^{\circ} \mathrm{C}$ дискриминанты РИС $M_{\mathrm{Cr}}, M_{\mathrm{Ni}}, M_{\mathrm{Fe}}$ соответственно равны $M_{\mathrm{Cr}}=3.89 \cdot 10^{9}>0, \quad M_{\mathrm{Ni}}=$ $=-1.9 \cdot 10^{9}<0, M_{\mathrm{Fe}}=-1.98 \cdot 10^{9}<0$. В данной работе при $T=350^{\circ} \mathrm{C}$ в согласии со знаками дискриминантов $M_{\mathrm{Cr}}, M_{\mathrm{Ni}}, M_{\mathrm{Fe}}$ наблюдается обеднение по $\mathrm{Cr}$ и обогащение по $\mathrm{Ni}$ и $\mathrm{Fe}$ при дозах облучения, близких к дозе выхода на стационарный режим (см. рис. 5).

\section{4. Заключение}

В данной работе на основе модели, базирующейся на первом и втором законах Фика и учитывающей обратный эффект Киркендалла, проведено компьютерное моделирование РИС в сплаве $\mathrm{Fe}-20 \% \mathrm{Cr}-8 \% \mathrm{Ni}$. Для данного сплава рассчитаны зависимости концентраций $\mathrm{Cr}$ и $\mathrm{Ni}$ от температуры, скорости набора дозы, плотности дислокаций, дозы облучения. Приведены также концентрационные профили вакансий и межузельных атомов при различных температурах, плотностях дислокаций, скоростях набора дозы. Проведенный анализ чувствительности модели указывает на то, что концентрации $\mathrm{Cr}$ и $\mathrm{Ni}$ существенно зависят от энергий миграции соответственно вакансий $\mathrm{Cr}$ и $\mathrm{Ni}$ и энергии миграции вакансий $\mathrm{Fe}$. Полученные значения концентраций $\mathrm{Cr}$ и Ni при дозах облучения близких к дозе выхода на стационарный режим находятся в согласии со знаками дискриминантов РИС для этих элементов $M_{\mathrm{Cr}}, M_{\mathrm{Ni}}$.

\section{Финансирование работы}

Работа выполнена за счет средств бюджетной программы Украины КПКВК 6541230 „Поддержка развития приоритетных направлений научных исследований“.

\section{Конфликт интересов}

Авторы заявляют, что у них нет конфликта интересов.

\section{Список литературы}

[1] G.S. Was. Fundamentals of Radiation Materials Science: Metals and Alloys. Springer-Verlag, Berlin (2007). 828 p.

[2] В.Н. Воеводин, И.М. Неклюдов. Эволюция структурнофазового состояния и радиационная стойкость конструкционных материалов. Наук. думка, Киев (2006). 376 с.

[3] A. Aitkaliyeva, L. He, H. Wen, B. Miller, X.M. Bai, T. Allen. In Woodhead Publishing Series in Energy: N.106. Structural Materials for Generation IV Nuclear Reactors / Ed. P. Yvon. Elsevier Ltd., Amsterdam (2017). P. 253-283.

[4] К.В. Чуистов. Старение металлических сплавов. 2-е изд. Академпериодика, Киев (2003). 567 с.

[5] O.V. Koropov, R.V. Skorokhod. East Eur. J. Phys. 1, 75 (2019). 
[6] T.R. Allen, G.S. Was. Acta Mater. 46, 3679 (1998).

[7] O.V. Koropov. Proc. of Eighteenth International Scientific Mykhailo Kravchuk Conference, October 7-10, 2017, Kyiv: V. 1. NTUU „KPI“, Kyiv (2017). P. 86.

[8] F. Soisson. J. Nucl. Mater. 349, 235 (2006).

[9] K. Vörtler, M. Mamivand, L. Barnard, I. Szlufarska, F.A. Garner, D. Morgan. J. Nucl. Mater. 479, 23 (2016).

[10] K.G. Field, Y. Yang, T.R. Allen, J.T. Busby. Acta Mater. 89, 438 (2015).

[11] H.-E. Schaefer, K. Maier, M. Weller, D. Herlach, A. Seeger. J. Diehl. Scripta Metallurgica 11, 803 (1977).

[12] P. Olsson, C. Domain, J. Wallenius. Phys. Rev. B 75, 014110 (2007).

[13] R.V. Skorokhod, O.M. Buhay, V.M. Bilyk, V.L. Denysenko, O.V. Koropov. East Eur. J. Phys. 5, 1, 61 (2018).

[14] S. Watanabe, H. Takahashi. J. Nucl. Mater. 208, 191 (1994).

Редактор Т.Н. Василевская 